\title{
Progress in freeform mirror design for space applications
}

\author{
Jerome Caron, Stefan Bäumer
}

Jerome Caron, Stefan Bäumer, "Progress in freeform mirror design for space applications," Proc. SPIE 11852, International Conference on Space Optics ICSO 2020, 118521S (11 June 2021); doi: 10.1117/12.2599322

SPIE Event: International Conference on Space Optics - ICSO 2021, 2021, Online Only 


\section{International Conference on Space Optics-ICSO 2020}

Virtual Conference

30 March-2 April 2021

Edited by Bruno Cugny, Zoran Sodnik, and Nikos Karafolas
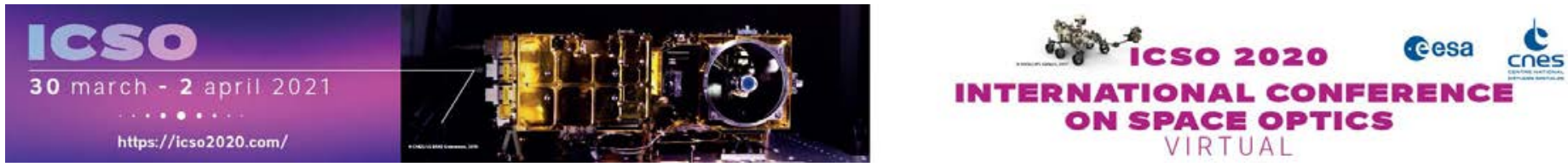

\section{Progress in freeform mirror design for space applications}

\section{Cesa isoporeseatings denes}




\title{
Progress in freeform mirror design for space applications
}

\author{
Jérôme Caron*a, Stefan Bäumer ${ }^{\mathrm{a}}$ \\ ${ }^{a}$ TNO, Optics Department, Stieltjesweg 1, 2628 CK Delft, The Netherlands
}

\begin{abstract}
Off-axis systems with freeform mirrors is a design approach of increasing importance for space instruments. While the off-axis reflective approach allows simple, versatile and obstruction free designs, the use of freeform surfaces allows to achieve better optical performance and/or compactness. In recent years, many such instruments have been designed and manufactured at TNO. Some important examples are the Tropomi (S5/precursor) and TSBOA (Sentinel-5) telescopes and several pushbroom spectrometers of the Spectrolite family.

Despite the recognized potential of these systems, there is to our knowledge no available theory that allows to describe and predict the aberrations of plane symmetrical (off-axis) systems with freeform mirrors. In this context, an effort has been started 4 years ago at TNO to develop an approach that can describe and explore systematically off-axis freeform mirror systems with an arbitrary geometry. Since then the developed theory has been proven to be very useful in scanning the solution space for better starting designs, folding geometries and correction of lower aberrations in an early design phase. To circumvent difficulties linked to a wavefront formalism, generalized ray-tracing equations were derived, that include aberration terms up to $3^{\text {rd }}$ order in $\mathrm{X} / \mathrm{Y}$ object and pupil coordinates. These equations were recently published in two papers for the case of pure mirror systems [1,2]. The theory was also expanded to describe flat reflective gratings, opening a way to a complete description of reflective freeform spectrometers.

In the present paper, after providing a high-level description and introduction to the aberration theory for freeform mirror systems, we will report some of its outcomes that have some practical relevance for space instruments. In particular:

- two-mirror telescope designs for slit spectrometers (thus having a 1D field along the slit) that are inherently corrected for spatial smile will be presented. For these designs the slit projection in object space is exactly straight.

- a new family of mirror spectrometers will be introduced that uses flat gratings and no collimator. In a collimator-less spectrometer, the aberrations induced by the grating under diverging light are corrected with freeform mirrors. The presented designs are entirely calculated from theory, with only optimization of the higher order freeform terms. A simpler architecture than traditional designs is obtained, with less optical surfaces.

- finally we present a systematic classification of distortions in imaging slit spectrometers. The difference between distortions originating in the collimator and at the grating or in the imager is clarified and described mathematically. We discuss aberration-induced non-linear dispersion, as well as distortions from the keystone and smile families. The proposed classification also applies to catadioptric and refractive systems, the only requirement being to have a plane of symmetry.
\end{abstract}

Keywords: freeform, freeform mirror, optical design, small satellites.

\section{OVERVIEW OF FREEFORM MIRROR PROJECTS AT TNO}

In this first section, we provide an overview of the main space instrument projects from the past 2 years where TNO was designing and/or manufacturing systems with freeform mirrors. The largest fraction of TNO optical instrument projects are for the space and semi-conductor markets, and within these two markets freeform optics are most often used for space designs. The following instruments therefore represents a good snapshot of our freeform mirror design work.

\subsection{Sentinel-5 telescope TSBOA}

Sentinel-5 is part of the European Earth Observation program Copernicus. It is a pushbroom spectrometer covering 7 bands (UV-1 and -2, VIS, NIR-1 and -2, SWIR-1 and -3) that will be placed on Metop-SG-A to monitor air quality and

*jerome.caron@tno.nl 
investigate atmosphere composition-climate interaction, with the main measured products being $\mathrm{O}_{3}, \mathrm{NO}_{2}, \mathrm{SO}_{2}, \mathrm{HCHO}_{\text {, }}$ $\mathrm{CHOCHO}$ and aerosols. Under the industrial prime contractor Airbus Defence and Space GmbH Germany, TNO is in charge of the design and manufacturing of the telescope assemblies as well as the UV1 spectrometer. TNO is in the core team and also designs and builds part of the Optical Ground Support Equipment (OGSE). The project is ongoing since several years and well advanced in the manufacturing phase.

The Sentinel-5 telescope is called TSBOA (Telescope, Scrambler and Beam splitter Optical Assembly) and images the Earth onto the entrance slit of the S5 spectrometers. Two identical telescopes that differ only by their coatings, are used on one hand for the UV1-SWIR1-SWIR3 spectrometers (US telescope) and on the other hand for the UV2, VIS, NIR1 and NIR2 spectrometers (VN telescope). The telescope has a field of view of $0.5 \mathrm{deg}$ in the along-track direction, and $109 \mathrm{deg}$ in across-track. It consists of two positive freeform mirrors, with a pupil plane located in between the mirrors. The pupil plane is also the plane where the polarization scrambler is located. This scrambler ensures that the efficiency of the whole system is independent of the incoming polarization state.

The optical design of TSBOA telescope is the same as for Tropomi [3]. The main optical differences between TSBOA and Tropomi are the use of different mirror coatings, a slit homogenizer rather than a regular slit and a different polarization scrambler design.

The TSBOA VN proto flight model system was delivered to Airbus in Q4 2020. The US TSBOA will be delivered in 2021. Four more flight models (two TSBOA VN and two TSBOA US) will be delivered in the future, for a total of three Sentinel-5 systems.
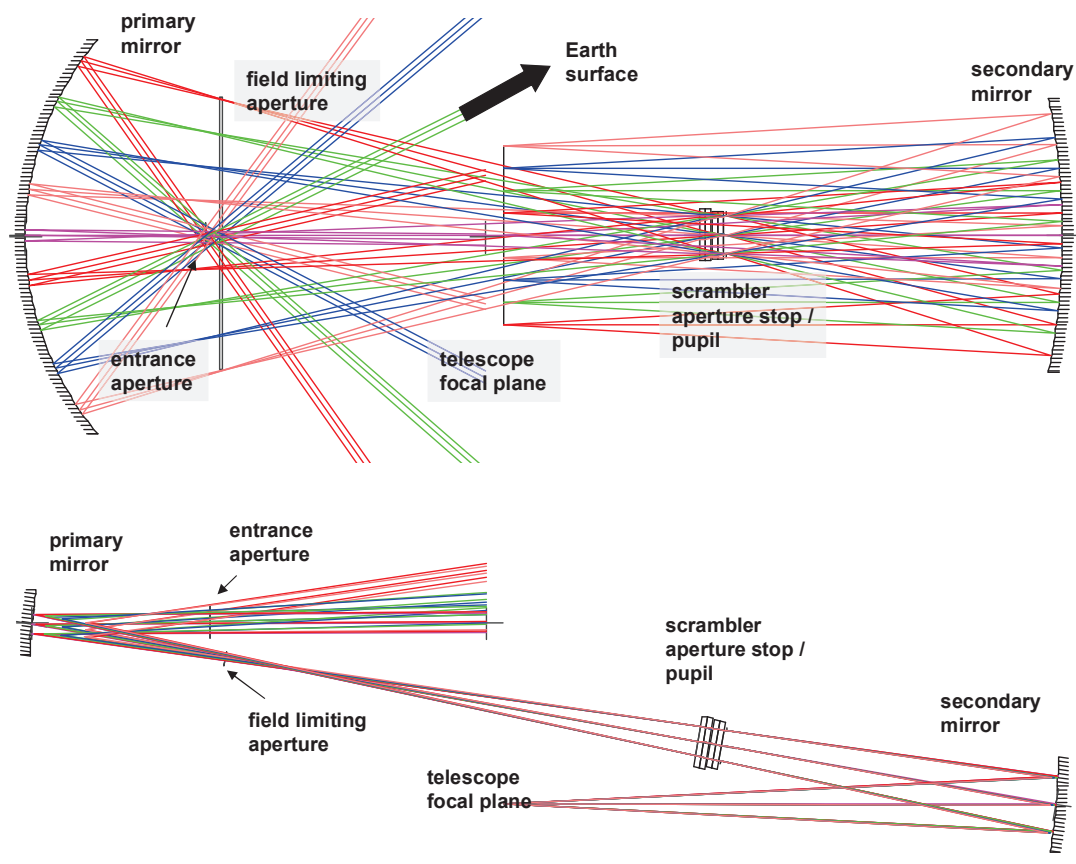

Figure 1. TSBOA telescope. The two mirrors have a freeform surface shape.

\subsection{Sentinel-5 UV1 spectrometer}

The Sentinel-5 UV1 spectrometer covers the spectral range $270-310 \mathrm{~nm}$ with a slit with dimensions $65 \mathrm{~mm} * 0.48 \mathrm{~mm}$, and a F/9*F/10 beam. Due to the inherent low radiance in this band, the UV1 spectrometer has very challenging specifications on in-band and out-of band straylight, as well as for keystone and frown. Achieving a low polarization sensitivity was also a challenge. 
The design is based on a modified Offner spectrometer with magnification 0.4 . The collimator and imager mirrors are both freeform and the grating is an off-axis asphere. The field lens (Frown Straylight Correction Plate, FSCP) is also a freeform component with the dual function to correct frown and suppress spectral straylight with a graded filter. The design and assembly were a joined effort between Airbus and TNO. The grating was made by Zeiss, the mirrors by AMOS/Balzers and the FSCP by Optimax/Balzers. The first proto flight model of the UV1 spectrometer was delivered to Airbus in Q3 2020. More details about the Sentinel-5 UV1 spectrometer can be found in [4] or in the following paper that will also be presented at ICSO 2020 [5].

\subsection{CHAPS-D}

CHAPS-D is a demonstrator for the CHAPS instrument (Compact Hyperspectral Air Pollution Sensor), a NASA project where the John Hopkins University Applied Physics Laboratory (APL) is industrial prime contractor, and TNO is in charge of the optical design. Although designed with requirements for a spaceborne instrument, CHAPS-D will first be flown on-board an aircraft. Upon a successful airborne demonstration, CHAPS-D will be then prepared for space flight. The instrument is a radiometrically calibrated hyperspectral imager operating in the spectral range 300-500 nm at a resolution of $0.6 \mathrm{~nm}$, and covering a swath of $100 \mathrm{~km}$ with GSD of $1 \mathrm{~km} * 1 \mathrm{~km}$ from an altitude of $500 \mathrm{~km}$. The design configuration is purely reflective and uses freeform mirrors, based on the TNO heritage of spectrometers. It consists of a two-mirror telescope, a single mirror collimator, a reflective grating and a three-mirror imager. All mirrors are freeform and the whole system is designed to be as small as possible to fit on a $6 \mathrm{U}$ CubeSat. At the time of writing this paper, PDR (preliminary design review) was successfully passed.

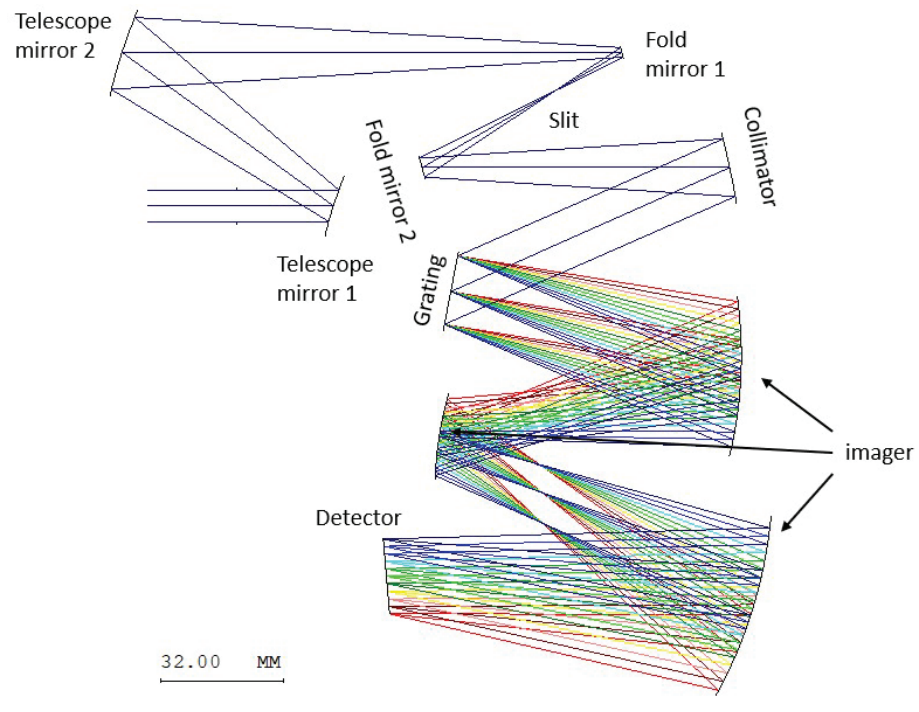

Figure 2. CHAPS-D optical design. All powered mirrors have a freeform surface shape.

\subsection{Wide FOV telescope for multibeam laser communication}

A reflective system based entirely on freeform mirrors was also designed for a laser satellite communication application. The instrument is intended to be operated with a spectral band around $1550 \mathrm{~nm}$ for multi-beam laser communication between Earth and a GEO satellite. It aims to provide coverage of an area about the size of Europe from GEO, serving as a link to multiple communication terminals (ground, ships or airplanes). It is a three-mirror F/5.8 telescope with an entrance pupil diameter of $200 \mathrm{~mm}$, a field of view of $8^{\circ} \times 3.2^{\circ}$ and a RMS WFE smaller than $50 \mathrm{~nm}$ [6].

These design requirements are very challenging. A first solution was obtained using traditional freeform design scripts already used for spaceborne instruments. The WFE was further decreased and the FOV increased with a careful and systematic optimization. On the other hand, several solutions were also obtained starting from a dummy design with flat 
mirrors, using a CMA-ES optimization tool (Covariance Matrix Adaptation Evolutionary Strategy) interfaced to the CodeV software [7]. One of the most interesting solutions obtained with the optimization tool was very close to the design obtained with the traditional approach, and is presented below on figure 3. This work was performed as part of TNO Early Research Program (ERP).

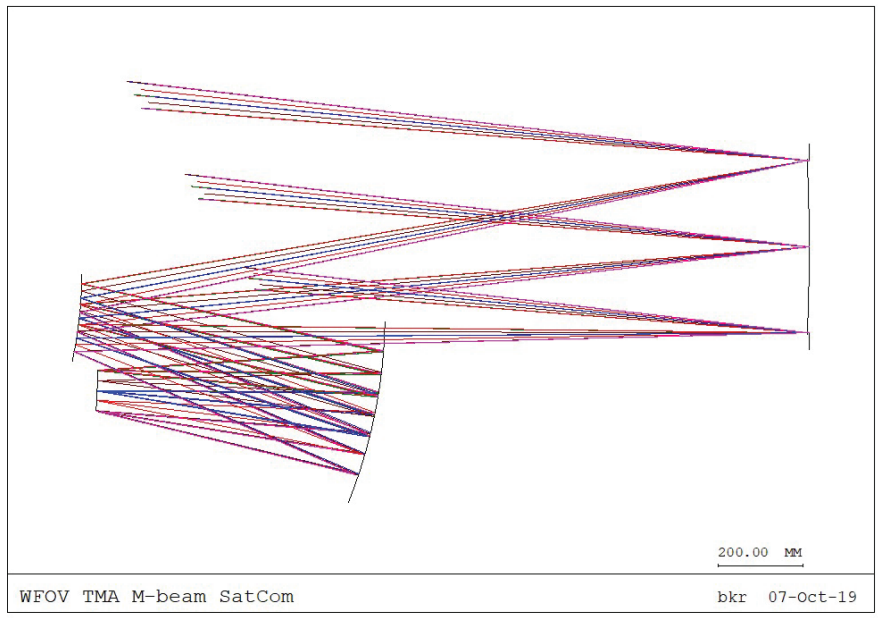

Figure 3. Laser satellite communication multibeam telescope. The three mirrors have a freeform surface shape.

\subsection{TANGO}

The Twin ANthropogenic Greenhouse Gas Observers (TANGO) mission comprises two agile satellites, TANGO-Carbon and TANGO-Nitro, each carrying a compact spectrometer from the TNO Spectrolite family. TANGO will monitor and quantify emissions of the greenhouse gases methane $\left(\mathrm{CH}_{4}\right)$ and carbon dioxide $\left(\mathrm{CO}_{2}\right)$ at the level of individual industrial facilities and power plants. The mission has been proposed to ESA as part of the ESA-Scout program by a consortium consisting of Innovative Solutions in Space (ISISpace), TNO, SRON and KNMI. TNO is in charge of the design and manufacturing of the two instruments, SRON and KNMI have the scientific lead, and ISISpace is the prime contractor and will provide the platform.

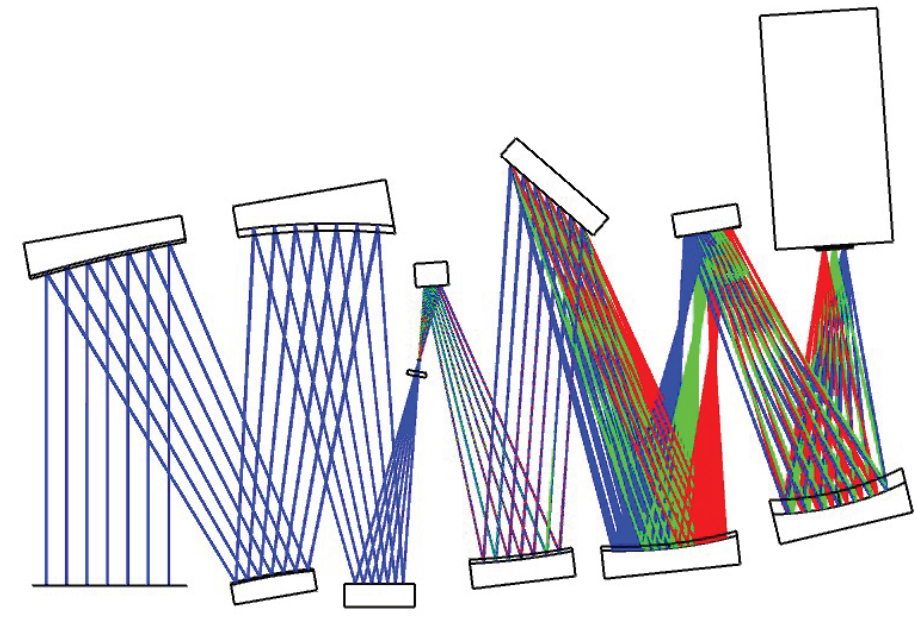

Figure 4. Tango-Carbon optical design. From left to right: we first find the telescope that consists of a TMA, then two flat folding mirrors are used on both sides of the slit, and finally the spectrometer which consists of a one freeform mirror collimator, a flat grating and a three freeform mirror imager. 
Both TANGO instruments cover a $30 \mathrm{~km}$ swath from a $500 \mathrm{~km}$ altitude, with a ground sampling distance of $300 \mathrm{~m}$. The spectral ranges are 405-490 nm (Nitro), and 1590-1675 nm (Carbon). The apertures are 50x38 mm (Nitro); and 64x60 $\mathrm{mm}$ (Carbon). Both designs are reflective pushbroom spectrometers. The telescopes are TMAs (off-axis conics), and freeform collimator and imager mirrors are used in the spectrometer. Both designs fit in an $8 \mathrm{U}$ instrument volume on a $16 \mathrm{U}$ small satellite platform. Fitting the large-aperture instruments in the small volume was a significant design challenge.

\subsection{Acknowledgements}

The freeform optical design work that is reported in this first part has been done by several designers, with contributions from Bob Kruizinga and David Nijkerk (TSBOA, Laser sat com Wide FOV), James Day (Sentinel-5 UV1, TANGO) and Rob Vink (TSBOA, Sentinel-5 UV1, CHAPS-D).

\section{ABERRATION THEORY FOR FREEFORM MIRROR SYSTEMS}

\subsection{Overview}

In this section we summarize the main aspects of the aberration theory developed at TNO for freeform mirror systems. More details can be found in two recently published papers [1,2].

Despite the recognized potential of off-axis freeform mirror systems, there is to our knowledge no available theory that describes systematically their aberrations prior to our work. The classical works on multi-mirror systems [8, 9] are restricted to rotationally symmetric designs with a decentered field and/or pupil and only traditional surfaces (spheres or conics). Some more recent works provide a description of the aberrations in more general systems but unfortunately without allowing quantitative predictions with explicit formulas. This is the case of the Nodal Aberration Theory (NAT) developed by Shack and Thompson [10] and recently extended to freeform surfaces by Fuerschbach [11]; or of the description and classification of aberrations by Sasian [12]. Other works are restricted either to non-freeform surfaces and/or do not account for paraxial astigmatism (in multi-mirror systems, intermediate images may have different locations in the meridional and sagittal planes).

To develop an aberration theory we chose to consider optical systems with the following characteristics:

- Anamorphic systems, with a different focal length or magnification in X and Y. Anamorphic systems are ubiquitous in space borne hyperspectral imagers that require different magnification along the spatial and spectral axes. They rely on cylindrical or toroidal optical components, that create intermediate images with paraxial astigmatism (a different location of the tangential and sagittal images). Calculating the aberrations of systems with paraxially astigmatism is a significant complication. As we will see in section 2.2 , there is no exact astigmatic wavefront and therefore no clear reference for computing wave aberrations in such systems. This is one of the reasons why it was chosen early on to develop a formalism based on ray-tracing equations.

- Systems with a plane of symmetry (called in short "off-axis"). Such systems have new aberration terms as compared to rotationally symmetric systems. The most dramatic change is the appearance of aberrations of lower order. While rotationally symmetric systems suffer from classical $3^{\text {rd }}$ order transverse aberrations, planesymmetrical systems have additionally $2^{\text {nd }}$ order transverse aberrations (constant coma, linear astigmatism and quadratic distortions). The adjectives "constant", "linear", "quadratic" and "cubic" refer to the dependency of the aberrations with field coordinates.

- Systems with freeform mirrors. The mirrors can take any arbitrary shape as long as it fulfills the planar symmetry (fully asymmetrical mirror shapes are not necessary, they will only introduce additional aberrations and degrade performance). While the freeform shapes can be specified in a number of ways: Legendre, Chebyshev, Zernike or Forbes polynomials, NURBS, etc., we chose to specify the shape as a simple XY polynomial that is well suited to a Seidel-like theory of aberrations based on power expansions, also knowing that any other surface specification can be expanded as a XY polynomial.

To describe such systems, we identify a ray as a 4-elements vector component defined with 2 positions $\left(h_{X}\right.$ and $\left.h_{Y}\right)$ and 2 angle tangents $\left(\tau_{X}=\tan \left(u_{X}\right)\right.$ and $\left.\tau_{Y}=\tan \left(u_{Y}\right)\right)$, where $u_{X}$ and $u_{Y}$ are the angles between the ray and the optical axis, after 
projection on the $(\mathrm{X}, \mathrm{Z})$ and $(\mathrm{Y}, \mathrm{Z})$ planes. The tangents of the angles can be linked to the direction cosines (L,M,N) used in optical design software with:

$$
\left(\begin{array}{c}
L \\
M \\
N
\end{array}\right)=\frac{1}{\sqrt{1+\tau_{X}^{2}+\tau_{Y}^{2}}}\left(\begin{array}{c}
\tau_{X} \\
\tau_{Y} \\
1
\end{array}\right)
$$

An important result that was obtained is a set of generalized ray-tracing equations to propagate the 4-element ray vector through an N-mirror system including aberration terms up to $3^{\text {rd }}$ order (transverse aberrations of $3^{\text {rd }}$ order in field and pupil coordinates). Two sets of such formulas have been derived: one for free-space propagation, and one for mirror reflection. See respectively equations (3) and (30-33) in reference [1]. It is worth noting that these formulas include both intrinsic aberrations (created by the mirrors) and extrinsic aberrations (also called induced aberrations, which are the $3^{\text {rd }}$ order aberrations that appear when the incoming beam is already aberrated). Based on these formulas it was possible to isolate the aberration contribution of individual mirrors and provide closed-form equations that are well suited for computing the aberrations in complex multi-mirror systems.

For the design of space optical instruments, these closed-form equations allow a quick identification of special solutions that correct the low-order aberrations. Such solutions are better starting designs for optimization and allow to reduce the dimensionality of the optimization problems. The insight that is gained about aberration correction also allows to identify suitable degrees of freedom to achieve a certain condition or performance (e.g. correction of distortions like smile, keystone). This aberration theory is powerful, as it leads not only to analytical equations but also simple numerical procedures to model off-axis freeform mirror systems.
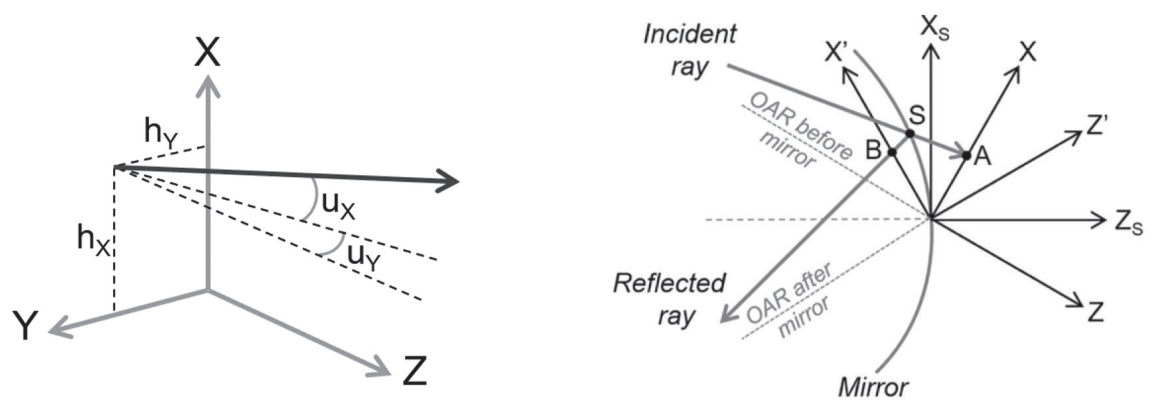

Figure 5 (left). A ray is defined by its four coordinates $h_{X}, h_{Y}, \tau_{X}=\tan \left(u_{X}\right), \tau_{Y}=\tan \left(u_{Y}\right)$.

Figure 6 (right). Geometry for the reflection on a freeform mirror surface. $(X, Y, Z)$ is the coordinate system for the incoming beam, and $\left(X^{\prime}, Y^{\prime}, Z^{\prime}\right)$ for the reflected beam. The mirror coordinate system is $\left(X_{S}, Y_{S}, Z_{S}\right)$. The ray propagation is typically computed with 3 steps. First, a free-space propagation of the incoming ray that ends at $A$ in the $(X, Y, Z)$ system. Then, the reflection transfers the point $A$ to point $B$ in the ( $\left.X^{\prime}, Y^{\prime}, Z^{\prime}\right)$ system. Finally a free-space propagation will start from $B$. OAR means "optical axis ray" and is used as a reference to measure ray heights and angles.

\subsection{Non-existence of the exact astigmatic wave}

One important discovery in the development of the freeform aberration theory was the realization that exact astigmatic waves that converge to two orthogonal focal lines do not exist. This result was also demonstrated independently by Tessmer [13]. In references [1,2] we provide a more general proof and a description of the intrinsic aberrations of an astigmatic wave. In this section we will illustrate our findings with a practical case.

A simple way to check experimentally the non-existence of an exact astigmatic wave is to set up a simple toroidal mirror in an optical design software package (Zemax, CodeV). Using XY polynomial surfaces one can define a toroidal parabola with additional freeform terms. The toroidal parabola creates some paraxial astigmatism with two different image planes. The freeform terms will be used to manipulate the wavefront that exits the parabola after reflection and modify it in all possible manners, trying to correct the observed aberrations. 
The shape of our toroidal parabola is described with:

$$
S(x, y)=\frac{x^{2}}{2 R_{X}}+\frac{y^{2}}{2 R_{Y}}+C x^{4}+D x^{2} y^{2}+E y^{4}
$$

The design is illustrated below. We use $\mathrm{R}_{\mathrm{X}}=-200 \mathrm{~mm}, \mathrm{R}_{\mathrm{Y}}=-400 \mathrm{~mm}$ and a circular pupil with diameter $50 \mathrm{~mm}$. The focal lines are located respectively at $100 \mathrm{~mm}$ (X-focus) and $200 \mathrm{~mm}$ (Y-focus) from the mirror.

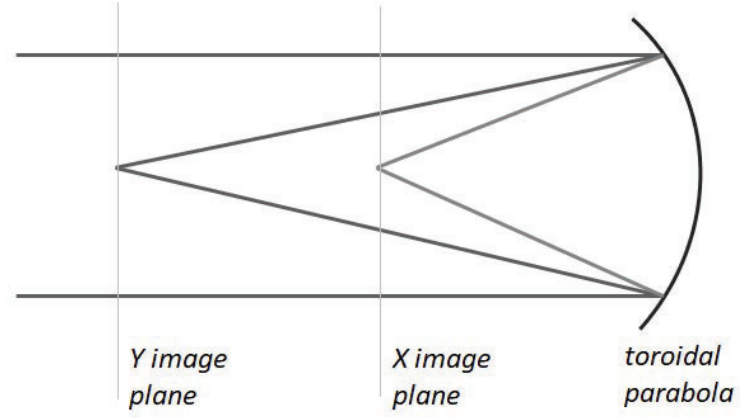

Figure 7. Toroidal parabola with $R_{X}=-200 \mathrm{~mm}$ and $R_{Y}=-400 \mathrm{~mm}$.

The freeform coefficients cover all possible terms that are compatible with the system planar symmetry. We start by setting all the coefficients to zero: $\mathrm{C}=\mathrm{D}=\mathrm{E}=0$. If we look carefully at the obtained focal lines, and if we enlarge the scale orthogonal to the lines by a factor $100 \mathrm{x}$ we see that the obtained focal lines are not perfectly sharp and suffer from aberrations.

If now, we set $\mathrm{C}=\mathrm{E}=0$ and the value of $\mathrm{D}$ equal to:

$$
D=D_{Y}=\frac{R_{X}-R_{Y}}{2 R_{X}^{2} R_{Y}^{2}}
$$

we obtain a focal line with twice as much aberrations at the $\mathrm{X}$ image plane, and a focal line that is nearly perfectly focused at the $\mathrm{Y}$ image plane. The opposite situation, with a corrected $\mathrm{X}$ focal line and twice as much aberration for the $\mathrm{Y}$ focal line is obtained for $\mathrm{D}=\mathrm{D}_{\mathrm{X}}=-\mathrm{D}_{\mathrm{Y}}$.

The values of $\mathrm{D}=\mathrm{D}_{\mathrm{X}}$ and $\mathrm{D}=\mathrm{D}_{\mathrm{Y}}$ can be calculated analytically with equation (3) or recovered with a software using a simple optimization. The other freeform coefficients $\mathrm{C}$ and $\mathrm{E}$ will not help to correct the focal lines and will only add more aberrations, degrading further image quality. This illustrates the fact that it is not possible to obtain simultaneously two perfect focal lines. The observed aberrations are $3^{\text {rd }}$ order in pupil coordinates, and correspond to a defocus that varies quadratically along the focal lines. It means that the focal lines are curved such that it is impossible to get a perfectly sharp focal line in a single image plane. When the D freeform coefficient is changed, the curvatures of both focal lines change together. It can be shown [2] that the following relationship holds:

$$
\frac{1}{\rho_{X}}-\frac{1}{\rho_{Y}}=\frac{2}{R_{Y}-R_{X}}
$$

where $\rho_{\mathrm{X}}$ and $\rho_{\mathrm{Y}}$ are respectively the radii of curvature of the focal lines at the $\mathrm{X}$ and $\mathrm{Y}$ image planes. This illustrates the impossibility to generate an exact astigmatic wave. More details and several proofs are provided in references $[1,2,13]$. 

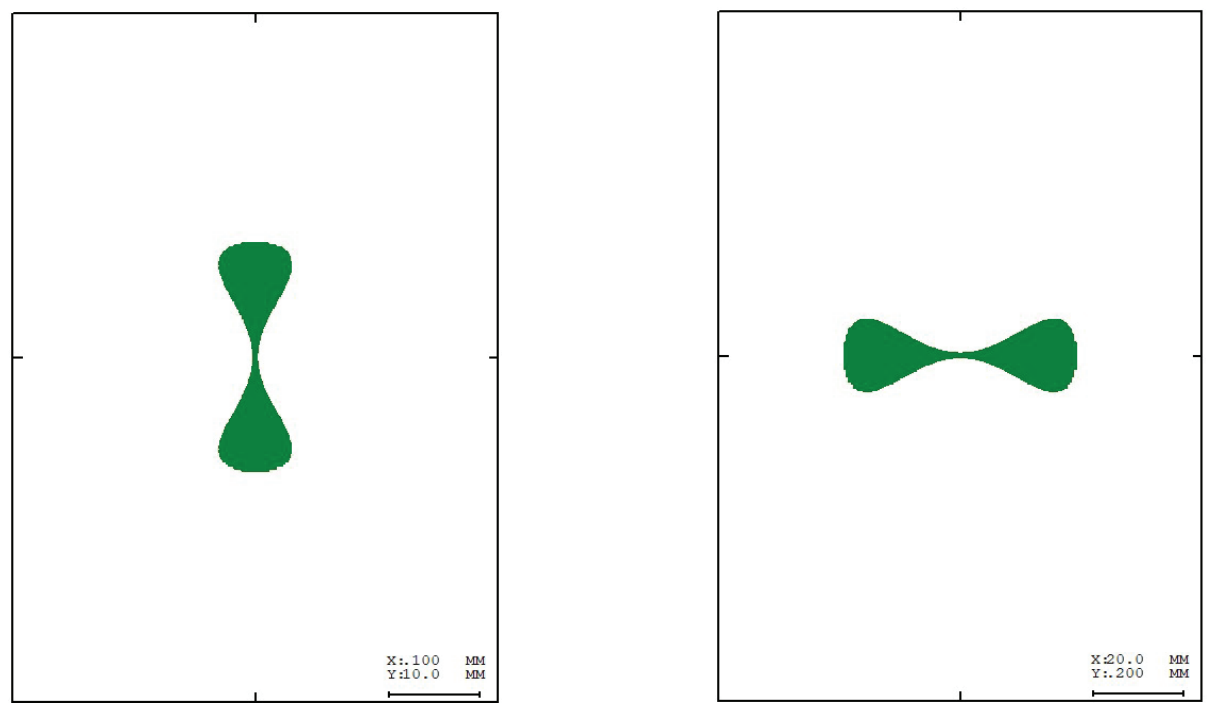

Figure 8. Focal lines obtained without any freeform term $(C=D=E=0)$. The observed aberrations are $3^{\text {rd }}$ order in pupil coordinates. Left: X image plane. The horizontal bar corresponds to $0.1 \mathrm{~mm}$ (horizontal) and $10.0 \mathrm{~mm}$ (vertical). Right: $Y$ image plane. The horizontal bar corresponds to $20.0 \mathrm{~mm}$ (horizontal) and $0.2 \mathrm{~mm}$ (vertical).
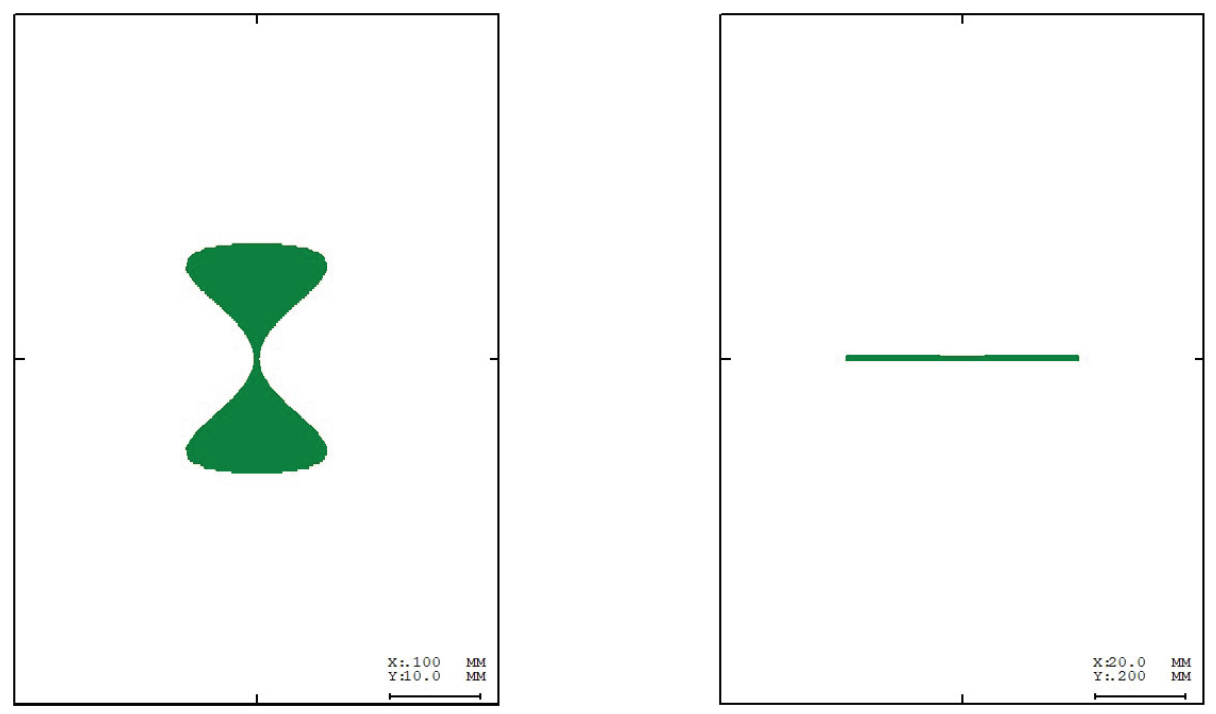

Figure 9. Same as figure 8, with $D=D_{Y}$. Aberrations at the $X$ focal plane are twice bigger. The focal line at the $Y$ focal plane is sharply focused. Left: X image plane. The horizontal bar corresponds to $0.1 \mathrm{~mm}$ (horizontal) and $10.0 \mathrm{~mm}$ (vertical). Right: Y image plane. The horizontal bar corresponds to $20.0 \mathrm{~mm}$ (horizontal) and $0.2 \mathrm{~mm}$ (vertical).

\subsection{Low-order aberrations}

To clarify the terminology we now provide a short description of the low-order aberrations. We reproduce below a table from reference [1], where all paraxial properties and $2^{\text {nd }}$ order transverse aberrations that can possibly exist in a system with a plane of symmetry are listed. The instrument plane of symmetry is $(X, Z)$ and for each term, the dependencies with respect to pupil $\left(\mathrm{h}_{\mathrm{X}}, \mathrm{h}_{\mathrm{Y}}\right)$ and object $\left(\mathrm{y}_{\mathrm{X}}, \mathrm{y}_{\mathrm{Y}}\right)$ coordinates are provided. 


\begin{tabular}{|c|c|c|c|c|c|}
\hline Group & Order & $\begin{array}{l}\text { Wavefront } \\
\text { disturbance }\end{array}$ & $\begin{array}{c}\text { Change of } \\
\text { transverse } \\
\text { coordinate } \delta \mathrm{X}\end{array}$ & $\begin{array}{c}\text { Change of } \\
\text { transverse } \\
\text { coordinate } \delta \mathrm{Y}\end{array}$ & Description \\
\hline \multirow{4}{*}{ 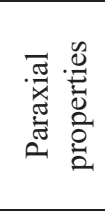 } & 1 & $h_{X}{ }^{2}+h_{Y}^{2}$ & $+\mathrm{h}_{\mathrm{X}}$ & $+\mathrm{h}_{\mathrm{Y}}$ & Power \\
\hline & 1 & $h_{X}^{2}-h_{Y}^{2}$ & $+\mathrm{h}_{\mathrm{X}}$ & $-h_{Y}$ & Paraxial astigmatism \\
\hline & 1 & $\mathrm{~h}_{\mathrm{xy}} \mathrm{x}$ & $\mathrm{yx}_{\mathrm{x}}$ & 0 & Magnification in $\mathrm{X}$ \\
\hline & 1 & $h_{Y y Y}$ & 0 & $\mathrm{y}_{\mathrm{Y}}$ & Magnification in $Y$ \\
\hline \multirow{8}{*}{ 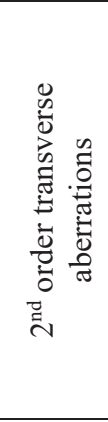 } & 2 & $\mathrm{hx}^{3}$ & $\mathrm{hx}^{2}$ & 0 & Constant coma 1 \\
\hline & 2 & $h_{X} h_{Y}{ }^{2}$ & $\mathrm{~h}_{\mathrm{Y}^{2}}$ & $\mathrm{~h}_{\mathrm{X}} \mathrm{h}_{\mathrm{Y}}$ & Constant coma 2 \\
\hline & 2 & $h_{x}^{2} y_{x}$ & $\mathrm{~h}_{\mathrm{x}} \mathrm{y}_{\mathrm{x}}$ & 0 & Linear astigmatism 1 \\
\hline & 2 & $h_{Y}^{2} y_{x}$ & 0 & $\mathrm{~h}_{\mathrm{Y}} \mathrm{yX}_{\mathrm{X}}$ & Linear astigmatism 2 \\
\hline & 2 & $h_{X} h_{Y} y_{Y}$ & $\mathrm{~h}_{\mathrm{Y} y \mathrm{Y}}$ & $\mathrm{h}_{\mathrm{X}} \mathrm{y}_{\mathrm{Y}}$ & Linear astigmatism 3 \\
\hline & 2 & $h_{x y x^{2}}$ & $\mathrm{yx}^{2}$ & 0 & Quadratic distortion 1 \\
\hline & 2 & $h_{X Y Y^{2}}$ & $\mathrm{yy}^{2}$ & 0 & Quad. dist. 2 (smile) \\
\hline & 2 & $\mathrm{~h}_{Y \mathrm{XXY}_{\mathrm{Y}}}$ & 0 & $\mathrm{yxy}_{\mathrm{X}}$ & Quad. dist. 3 (keystone) \\
\hline
\end{tabular}

Table 1. Paraxial properties and $2^{\text {nd }}$ order transverse aberrations in a general system (i.e. not only reflective) that has a plane of symmetry equal to $(X, Z)$. All terms that possibly exist given the instrument symmetry are mentioned. The dependencies with respect to pupil $\left(h_{X} h_{Y}\right)$ and object $\left(y_{X}, y_{Y}\right)$ coordinates is given.

In the first group with the paraxial properties, the power term has equal contributions from cylindrical powers in $\mathrm{X}$ and $\mathrm{Y}$, while the paraxial astigmatism has opposite contributions and will only be zero when both powers are equal. The magnification in $\mathrm{X}$ and $\mathrm{Y}$ are separate, meaning that the system may be anamorphic with different magnifications in $\mathrm{X}$ and $\mathrm{Y}$.

The constant coma has two contributions. Constant coma 1 is the tangential coma, while constant coma 2 is the sagittal coma. When the wavefront disturbances of both comas are equal they generate the familiar coma shape observed in rotationally symmetric systems, with a V-shaped caustic making an angle $60 \mathrm{deg}$. When they take different values, the shape of the aberrated spot changes (it may become more or less elongated with a different caustic angle, the caustic may disappear, or we may obtain only a line if the constant coma 2 is equal to zero).

The linear astigmatism has three terms (linear astigmatism 1, 2 and 3, abbreviated LA1, LA2 and LA3) which are rather difficult to illustrate. We will only describe them briefly. LA1 and LA2 generate focal lines that are aligned, respectively, along the $\mathrm{X}$ and $\mathrm{Y}$ axes of the image plane ; while LA3 generates focal lines at 45 deg between the $\mathrm{X}$ and $\mathrm{Y}$ axes. So far, we assumed that the image plane is orthogonal to the chief ray. If now we take $\mathrm{LA} 1=\mathrm{LA} 2 \neq 0$ and $\mathrm{LA} 3=0$ it is possible to recover a perfect image by tilting the image plane by the appropriate angle. It means that the sum of LA1 and LA2 with equal coefficients corresponds to a focus tilt, which is a $2^{\text {nd }}$ order transverse aberration. If the image plane may be tilted freely in the system (additional degree of freedom) then only 2 independent linear astigmatism terms needs to be corrected.

Finally, three types of quadratic distortions can occur in the system. They are illustrated on figure 10 below. The keystone (center of fig.10) and smile (right of fig.10) are familiar. The quadratic variation of magnification along X may have a similar magnitude as the smile and keystone and may also affect significantly the performance of an off-axis systems. Distortions are discussed further in section 3.2. 

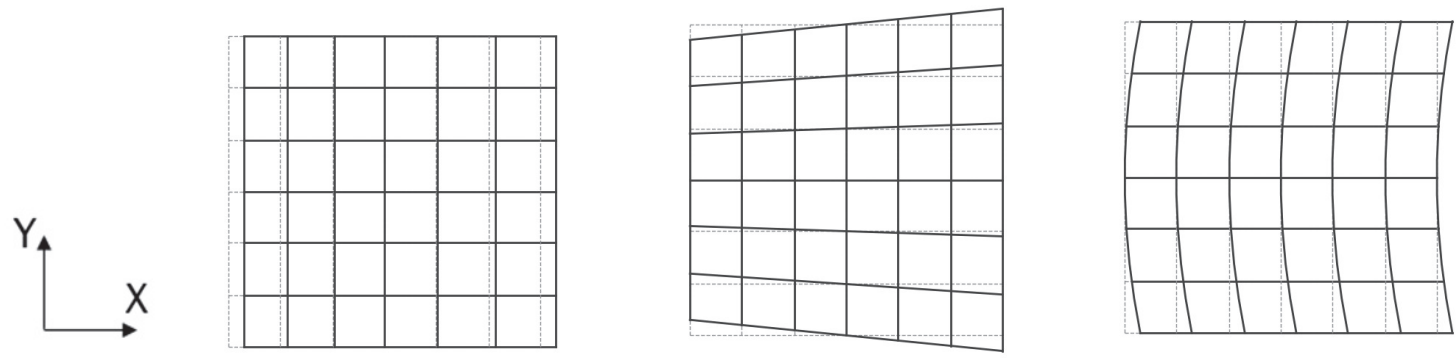

Figure 10. Quadratic distortions found in a system with a plane of symmetry (X,Z). Left: quadratic magnification. The distortion error $d X$ is proportional to $X^{2}$ and always keeps the same sign; on the figure $d X$ is positive. If we assume that $X=0$ at the center of the image plane, on the left part of the image (negative $X$ ) having $d X>0$ means moving towards $X=0$ so the interval between two adjacent vertical lines shrinks. On the right part of the image (positive X) it is the opposite and the interval between vertical lines increases. The spacing between two adjacent very close vertical lines is proportional to the derivative of $d X$ wrt $X$ and grows linearly across the figure. Center: keystone. Right: smile.

\section{SOME PRACTICAL OUTCOMES OF THE THEORY}

\subsection{Spatial smile free telescope for slit spectrometers}

Telescopes for pushbroom spectrometers typically project the entrance slit of a spectrometer in their object space (e.g. onto Earth surface). These telescopes have two peculiarities. First, since the image is the slit and is well-defined, distortion occurs in the object space. Additionally, due to the straight and very narrow slit shape, these telescopes will in practice not suffer from quadratic magnification or keystone. The only $2^{\text {nd }}$ order distortion that affects them is smile. In a pushbroom spectrometer, telescope smile is called "spatial smile" to differentiate it from spectrometer smile which occurs in the final image along the spectral axis.

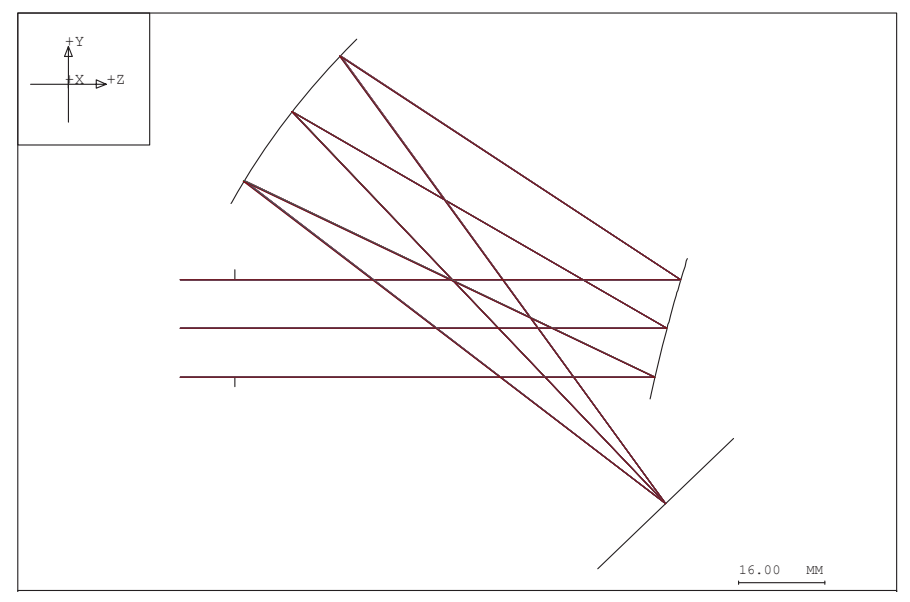

\begin{tabular}{|c|c|}
\hline \multicolumn{2}{|c|}{ Spatial smile free 2-mirror telescope } \\
\hline $\begin{array}{l}\text { Focal } \\
\text { lengths }\end{array}$ & $\begin{array}{c}\mathrm{f}_{\mathrm{X}}=64.5 \mathrm{~mm} \\
\mathrm{f}_{\mathrm{Y}}=255.7 \mathrm{~mm}\end{array}$ \\
\hline FOV & $7.28 \mathrm{deg}$ \\
\hline Spatial & $0.00034 \mathrm{deg}$ (no optim.) \\
\hline smile & $0.000036 \mathrm{deg}$ (after optim) \\
\hline
\end{tabular}

Figure 11. Two-mirror telescope free of spatial smile.

Table 2. Telescope parameters. 
Many pushbroom instruments suffer from a moderate spatial smile that is then considered acceptable. In some other cases, it is a feature that is undesirable and needs to be corrected at design level, prior to any Level-1 software correction. In the design of a two-mirror freeform telescope, correction of smile is challenging as it depends on the freeform mirror shapes as well as on the geometry and paraxial configuration chosen. For instance, if a system is designed and its geometry frozen, correction of spatial smile is usually impossible to achieve. Spatial smile correction has to be tackled early on in the design of such telescope. With the aberration theory we identified mathematically the sub-part of the design parameters space where spatial smile is inherently corrected. It is then possible to scan quickly through a family of solutions to converge to the most promising design. The same method may also be used to achieve a particular, nonzero value of the spatial smile if it is required for a particular application.

As an illustration, we present a two-mirror anamorphic freeform telescope with $f_{X}=64.5 \mathrm{~mm}, \mathrm{f}_{\mathrm{Y}}=255.7 \mathrm{~mm}$ (anamorphic ratio 4.0 ). The distances from entrance aperture to M1, from M1 to M2 and from M2 to the image plane are $80 \mathrm{~mm}, 80 \mathrm{~mm}$ and $100 \mathrm{~mm}$ respectively. The slit is $32 \mathrm{~mm}$ long. The full field of view is 7.28 deg in across-track and the RMS spot diameters are smaller than 12.0 microns for a rectangular entrance aperture $18 \mathrm{~mm} * 18 \mathrm{~mm}$. Distortions are independent from the entrance pupil dimensions. The paraxial configuration of this design was first calculated analytically and then entered in $\mathrm{CodeV}$ for final optimization. The spatial smile for the calculated configuration was $0.00034 \mathrm{deg}$ at the edge of the swath, while for an arbitrary layout the smile is usually comparable to 0.1 to $0.5 \mathrm{deg}$. After final optimization it was reduced by about a factor of 10 to $0.000036 \mathrm{deg}$, which corresponds to 0.15 arcsec so $0.4 \mathrm{~m}$ on Earth from a $600 \mathrm{~km}$ altitude.

\subsection{Systematic classification of spectrometer distortions}

If the distortions of a telescope for a slit-based spectrometer reduce to spatial smile, distortions in a slit spectrometer are more complex due to different contributions from the collimator and imager. In addition, higher order effects are often noticeable. We can define three coordinates in the final image of a slit spectrometer, assuming this image is perfect and has (yet) no distortions.

- $\quad\left(\mathrm{X}_{\text {slit }}, \mathrm{Y}_{\text {slit }}\right)$ are slit coordinates measured on the final image, spanning the slit image at a given wavelength: $\mathrm{X}_{\text {slit }}$ is along the width and is very small, $\mathrm{Y}_{\text {slit }}$ is along the length of the slit. $\mathrm{X}_{\text {slit }}$ and $\mathrm{Y}_{\text {slit }}$ are proportional to the coordinates measured on the real (physical) slit, after multiplication by the spectrometer magnifications $\mathrm{M}_{\mathrm{X}}$ and MY.

- $\mathrm{X}_{\lambda}$ is a coordinate than spans the entire final image along the spectral direction. It is proportional to the difference between the actual wavelength and the center wavelength, to the spectrometer angular dispersion and to the imager focal length. For instance, assuming a grating spectrometer, we can write the grating equation for the center wavelength $\lambda_{0}$, at the center of field and for the chief ray:

$$
\sin \alpha+\sin \beta=p \lambda_{0} N
$$

where $\alpha$ and $\beta$ are the incident and diffracted angles, $p$ is the diffraction order (equal to $\pm 1, \pm 2$, etc.) and $N$ the groove density $(1 / \mathrm{m})$. Then any deviation from the chief ray and center wavelength is quantified with small variations $\mathrm{d} \alpha, \mathrm{d} \beta$ and $\mathrm{d} \lambda$. By differentiation we find the approximate relationships:

$$
\begin{gathered}
X_{\lambda} \approx f_{\text {img }} d \beta=f_{\text {img }}\left(\frac{\sin \alpha+\sin \beta}{\cos \beta}\right) \frac{d \lambda}{\lambda_{0}} \\
X_{\text {slit }} \approx f_{\text {img }} d \beta=-f_{\text {img }} \frac{\cos \alpha}{\cos \beta} d \alpha
\end{gathered}
$$

Equations (6) and (7) shows that a change in angle $d \beta$ may have two contributions, one from a change of slit coordinate $\mathrm{X}_{\text {slit }} / \mathrm{M}_{\mathrm{X}}$ that results in $\mathrm{d} \alpha$, and the other from a change of wavelength $\mathrm{d} \lambda / \lambda_{0}$. After the dispersive component, the final coordinate along the $\mathrm{X}$ axis at the detector is the sum of these two contributions $\mathrm{X}_{\text {slit }}$ and $\mathrm{X}_{\lambda}$. In the same way, the final coordinate along the $\mathrm{Y}$ axis at the detector is simply equal to the $\mathrm{Y}$ slit coordinate: 


$$
\begin{gathered}
X_{d e t}=X_{\lambda}+X_{s l i t} \\
Y_{d e t}=Y_{s l i t}
\end{gathered}
$$

The distortions are small errors in positions along the $\mathrm{X}$ or $\mathrm{Y}$ directions. When generated in the collimator they will only depend on $\mathrm{X}_{\text {slit }}$ and $\mathrm{Y}_{\text {slit }}$; while distortions generated at the grating or prism, and in the imager will depend on the full coordinates $\mathrm{X}_{\text {det }}$ and $\mathrm{Y}_{\text {det. }}$. Distortions in a freeform system will take all possible dependencies that are allowed by the system symmetries. Since the instrument has a plane of symmetry that is orthogonal to the slit, distortions can only take the dependencies listed in table 3 . We will now group the distortions in different families, according to their dependency on $\mathrm{Y}_{\text {slit }}$ (last line in table 3).

\begin{tabular}{|l|c|c|}
\hline Dependency on... & Distortions along X & Distortions along Y \\
\hline $\begin{array}{l}\mathrm{X}_{\text {slit }} \text { (collimator) } \\
\text { or } \mathrm{X}_{\text {det }} \text { (prism/grating + imager) }\end{array}$ & Any & any \\
\hline $\mathrm{Y}_{\text {slit }}=\mathrm{Y}_{\text {det }}$ & $\begin{array}{c}\text { Even power } \\
\text { (constant, quadratic, } 4^{\text {th }} \text { power, etc.) }\end{array}$ & $\begin{array}{c}\text { Uneven power } \\
\text { (linear, cubic, } 5^{\text {th }} \text { power, etc) }\end{array}$ \\
\hline
\end{tabular}

Table 3. Allowed dependencies of the $X$ - and $Y$-distortions in a general (i.e. not only reflective) plane-parallel system.

\section{Non-linear dispersions}

This first distortion family contains the X-distortions that do not depend on $\mathrm{Y}_{\text {slit. }}$ The first and most important distortion is proportional to $\mathrm{X}^{2}$ and is a quadratic magnification. The distortion created in the collimator is proportional to $\mathrm{X}_{\text {slit }}{ }^{2}$ and is negligible since the slit is very narrow. When created by the prism/grating and the imager this distortion is proportional to $\mathrm{X}_{\mathrm{det}}{ }^{2}$, it is a quadratic magnification acting on the spectral coordinates. Due to this we call it "quadratic dispersion". The family also contains higher-order dispersions (cubic dispersion proportional to $\mathrm{X}^{3}$, etc.). Let's assume that the quadratic dispersion created in the imager is proportional to A. Ignoring effects with powers higher than 2 in $\mathrm{X}_{\text {slit }}$ we find:

$$
(d X)_{\substack{\text { quadratic } \\ \text { dispersion }}}=A X_{\text {det }}^{2} \approx A X_{\lambda}^{2}+2 A X_{\lambda} X_{\text {slit }}
$$

The second term in $\mathrm{X}_{\lambda} \mathrm{X}_{\text {slit }}$ tells us that the width of the slit image varies linearly across the spectral image. This is the effect that we observe on figure 12. This slit image widening is commonly observed in plane-parallel spectrometers. The numbers in table 4 were obtained with the preliminary design of a spectrometer. One sees the very large increase of the dispersion (in pixels $/ \mathrm{nm}$ ) with wavelength by almost a factor of 2 across the full spectral range, while the spectral resolution remains constant. With the same mechanism, the cubic dispersion $\mathrm{X}_{\lambda}{ }^{3}$ is associated with a term $3 \mathrm{X}_{\lambda}{ }^{2} \mathrm{X}_{\text {slit, }}$, which creates a quadratic variation of the slit width across the spectrum.

\section{Keystone family}

The Y-distortions that are linear in $\mathrm{Y}_{\text {slit }}$ belong to the keystone family. The main contributors are $\mathrm{XY}_{\text {slit }}$ (keystone) and $\mathrm{X}^{2} \mathrm{Y}_{\text {slit }}$ (quadratic keystone) with $\mathrm{X}=\mathrm{X}_{\text {slit }}$ or $\mathrm{X}_{\text {det }}$. The distortion is always proportional to $\mathrm{Y}_{\text {slit, }}$ so there is no distortion in the middle of the swath where $Y_{\text {slit }}=0$, and the distortion progressively increases as one goes to the edge of swath. The linear keystone $\mathrm{X}_{\lambda} \mathrm{Y}_{\text {slit }}$ is an important distortion, that one wants to correct to avoid co-registration errors (a detector line looking at different pixels on ground depending on the wavelength). When it is accurately corrected the residuals often show a quadratic contribution $\mathrm{X}_{\lambda}^{2} \mathrm{Y}_{\text {slit. }}$.

In the collimator, the keystone term $\mathrm{X}_{\text {slit }} \mathrm{Y}_{\text {slit }}$ creates a "slit keystone" that deforms all slit images in the same way. This effect has been observed in the SpexOne instrument [14] where specific requirements were defined to reduced it. It is illustrated on figure 13 (left). The linear and quadratic keystones that are generated at the grating/prism or in the imager 
impact the complete spectral image, as illustrated on figure 13 (center and right). One sees that the edges of the slit images on figures 13 (center and right) also become slightly tilted, in accordance to the shape of the image edge. This is due to an additional contribution to slit keystone that is created in the imager (second term in equation (11)). Let's assume that the linear keystone is equal to B. We find the following equation, where the first term is the linear keystone for the complete spectral image; and the second term is slit keystone and acts on each individual slit image.

$$
(d Y)_{\text {linear }}^{\text {keystone }}=B X_{\text {det }} Y_{\text {slit }}=B X_{\lambda} Y_{\text {slit }}+B X_{\text {slit }} Y_{\text {slit }}
$$

Quadratic and higher orders keystones are sometimes called "frown", with "keystone" reserved to the linear contribution.

\section{Smile family}

$\mathrm{X}$-distortions with even power $\left(2^{\text {nd }}, 4^{\text {th }}\right.$, etc) in $\mathrm{Y}_{\text {slit }}$ belong to the smile family. The traditional smile (figure 14, left) is proportional to $\mathrm{Y}_{\text {slit }}{ }^{2}$. It creates a parabolic curved slit image, and has contributions from all parts in the spectrometer: collimator as well as grating/prism and imager. While keystone generates non-correctable co-registration errors, smile is less harmful and makes the spectral calibration dependent on the position across the instrument swath. However it can create radiometric errors on non-uniform scenes [15].

A second important distortion is the linear variation of smile across the spectral image, that varies as $\mathrm{X}_{\text {det }} Y_{\text {slit }}{ }^{2}$. It is illustrated on figure 14 (center) and is described with the following equation:

$$
(d X)_{\substack{\text { variation of } \\ \text { smile }}}^{\text {sinear }}=C X_{\text {det }} Y_{\text {slit }}^{2}=C X_{\lambda} Y_{\text {slit }}^{2}+C X_{\text {slit }} Y_{\text {slit }}^{2}
$$

The second term (proportional to $\mathrm{X}_{\text {slit }}$ ) tells us that the smile also varies across one slit image, consistently with the smile variations in the complete spectral image. The linear variation of smile $X_{\operatorname{det}} Y_{\text {slit }}{ }^{2}$ often complicates greatly the effective correction of smile across a complete spectral image : the smile may be easily corrected at one wavelength but will not be corrected at other wavelengths. In theory, a linear variation of the smile across the slit can be also generated in the collimator (it is equal to $\mathrm{X}_{\text {slit }} \mathrm{Y}_{\text {slit }}{ }^{2}$ with a mechanism similar to the slit keystone: it acts on the slit and gives the same variation for all slit images) but it usually very weak and unnoticed. A $4^{\text {th }}$-order smile distortion (figure 14 , right) can sometimes be observed.

\section{Higher order Y-distortions}

For completeness we mention here higher-order distortions with uneven $\left(3^{\text {th }}, 5^{\text {th }}\right.$, etc.) power dependency on $Y_{\text {slit. }}$ These distortions are always very small.

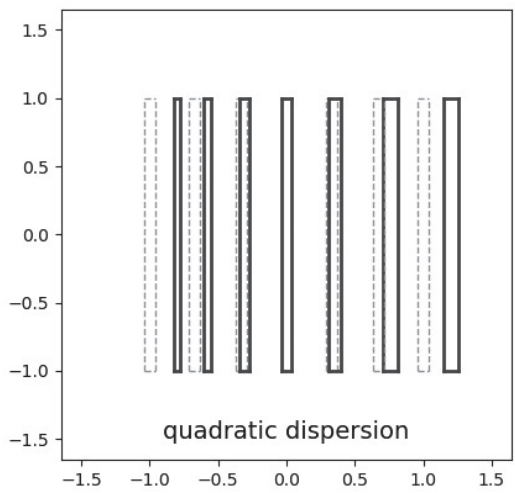

$\mathrm{nb}$ of pixels/nm
\begin{tabular}{|c|c|c|c|c|c|c|}
\hline X slit (mm) & Y slit (mm) & $770 \mathrm{~nm}$ & $673.75 \mathrm{~nm}$ & $577.5 \mathrm{~nm}$ & $481.25 \mathrm{~nm}$ & $385 \mathrm{~nm}$ \\
\hline 0 & 0 & 6.61 & 5.50 & 4.57 & 3.81 & 3.18 \\
3.2 & 0 & 6.62 & 5.51 & 4.58 & 3.81 & 3.18 \\
6.4 & 0 & 6.63 & 5.51 & 4.58 & 3.81 & 3.18 \\
9.6 & 0 & 6.63 & 5.51 & 4.58 & 3.82 & 3.18 \\
12.8 & 0 & 6.63 & 5.52 & 4.59 & 3.82 & 3.18 \\
\hline
\end{tabular}

spectral resolution (nm)
\begin{tabular}{|c|c|c|c|c|c|c|}
\hline X slit (mm) & Y slit (mm) & $770 \mathrm{~nm}$ & $673.75 \mathrm{~nm}$ & $577.5 \mathrm{~nm}$ & $481.25 \mathrm{~nm}$ & $385 \mathrm{~nm}$ \\
\hline 0 & 0 & 2.03 & 2.03 & 2.03 & 2.04 & 2.04 \\
3.2 & 0 & 2.02 & 2.02 & 2.02 & 2.02 & 2.02 \\
6.4 & 0 & 2.01 & 2.01 & 2.01 & 2.01 & 2.01 \\
9.6 & 0 & 2.00 & 2.00 & 2.00 & 2.00 & 2.01 \\
12.8 & 0 & 2.00 & 2.00 & 2.00 & 2.00 & 2.00 \\
\hline
\end{tabular}

Figure 12. Quadratic dispersion. The slit width also increases linearly across the spectral image.

Table 4. Linear dispersion and spectral resolution measured at the focal plane of an hyperspectral imager (a preliminary design was used). The linear dispersion increases by more than a factor $2 x$ across the spectral range; while spectral resolution is constant. 

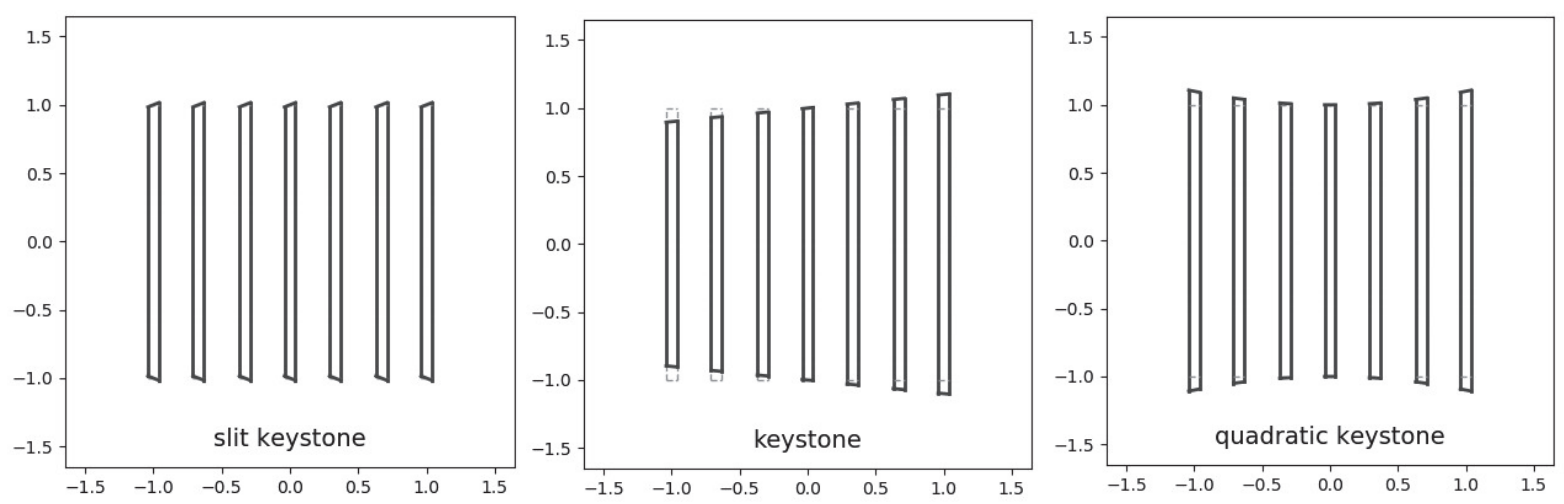

Figure 13. Distortions of the keystone family.
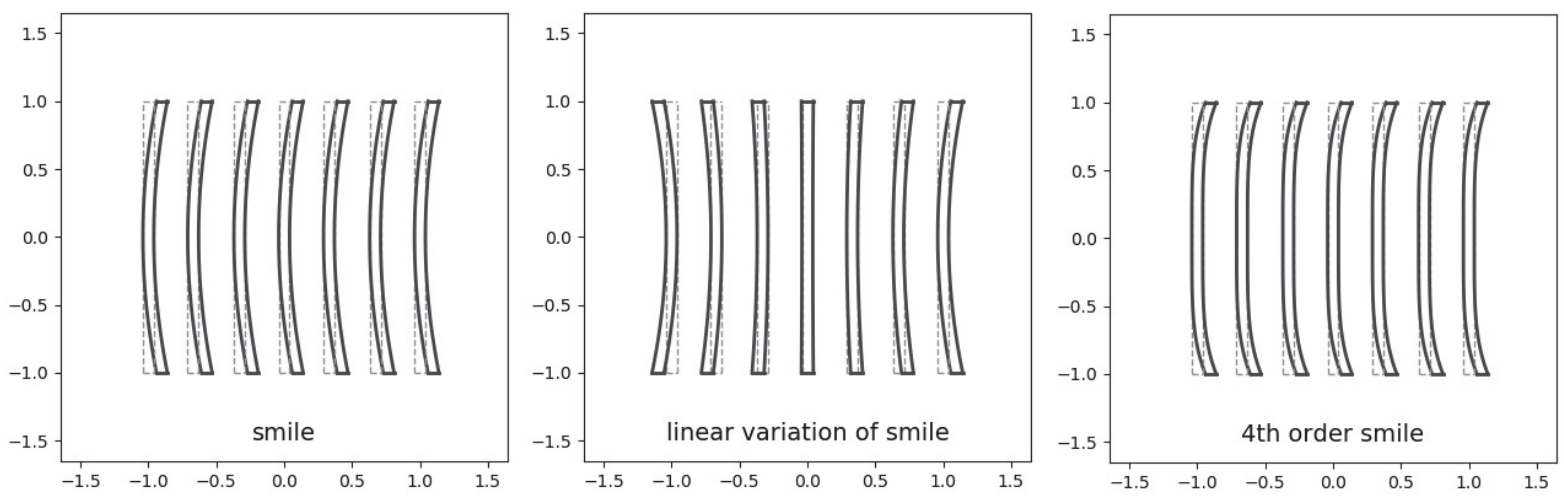

Figure 14. Distortions of the smile family.

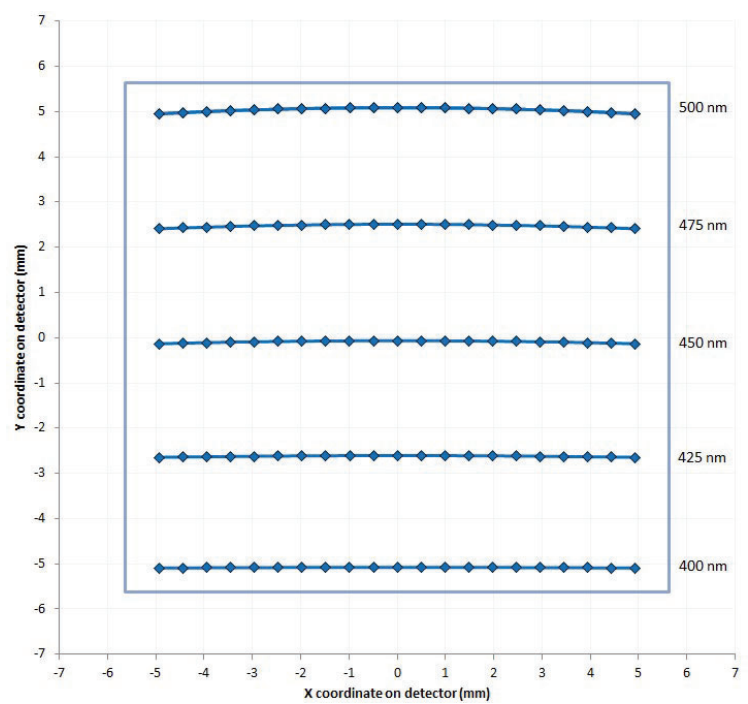

Figure 15. Focal plane of the Spectrolite spectrometer presented on fig. 18, showing almost perfect (sub-pixel) correction of keystone (keystone $=3.3 \mu \mathrm{m})$, and smile with a linear variation across the image (max. smile $=134.9 \mu \mathrm{m})$. 


\begin{tabular}{|c|c|c|c|}
\hline \multirow{2}{*}{$\begin{array}{l}\text { Non-linear } \\
\text { dispersions } \\
\text { (along } X \text { axis) }\end{array}$} & General form & $\left(X_{\lambda}\right)^{N}\left(X_{\text {slit }}\right)^{P}$ & with $\mathrm{N}+\mathrm{P} \geq 2$ \\
\hline & Main contributors & $\begin{array}{ll}\mathrm{X}_{\lambda}^{2} & 2 \mathrm{X}_{\lambda} \mathrm{X}_{\text {slit }} \\
\mathrm{X}_{\lambda}^{3} & 3 \mathrm{X}_{\lambda}^{2} \mathrm{X}_{\text {slit }} \\
\text { (etc.) } & \end{array}$ & $\begin{array}{l}\text { Quadratic distortion }(N, P)=(2,0) \text { and }(N, P)=(1,1) \text {. } \\
\text { Cubic distortion }(N, P)=(3,0) \text { and }(N, P)=(2,1) \text {. } \\
\text { Etc. }\end{array}$ \\
\hline \multirow{2}{*}{$\begin{array}{l}\text { Keystone } \\
\text { family } \\
\text { (along Y axis) }\end{array}$} & General form & $Y_{\text {slit }}\left(X_{\lambda}\right)^{N}\left(X_{\text {slit }}\right)^{P}$ & with $\mathrm{N}+\mathrm{P} \geq 1$ \\
\hline & Main contributors & $\begin{array}{ll}\mathrm{Y}_{\text {slit }} \mathrm{X}_{\lambda} & \mathrm{Y}_{\text {slit }} \mathrm{X}_{\text {slit }} \\
\mathrm{Y}_{\text {slit }} \mathrm{X}_{\lambda}^{2} & 2 \mathrm{Y}_{\text {slit }} \mathrm{X}_{\lambda} \mathrm{X}_{\text {slit }} \\
\mathrm{Y}_{\text {slit }} \mathrm{X}_{\lambda}^{3} & 3 \mathrm{Y}_{\text {slit }} \mathrm{X}_{\lambda}^{2} \mathrm{X}_{\text {slit }} \\
\text { (etc.) } & \\
\end{array}$ & $\begin{array}{l}\text { Linear keystone }(N, P)=(1,0) \text { and }(N, P)=(0,1) \text {. } \\
\text { Quadratic keystone }(N, P)=(2,0) \text { and }(N, P)=(1,1) \text {. } \\
\text { Cubic keystone }(N, P)=(3,0) \text { and }(N, P)=(2,1) \text {. } \\
\text { Etc. }\end{array}$ \\
\hline \multirow{4}{*}{$\begin{array}{l}\text { Smile family } \\
\text { (along } X \text { axis) }\end{array}$} & General form & $\left(\mathrm{Y}_{\text {slit }}\right)^{2 \mathrm{M}}\left(\mathrm{X}_{\lambda}\right)^{\mathrm{N}}\left(\mathrm{X}_{\text {slit }}\right)^{\mathrm{P}}$ & with $\mathrm{M}=1,2,3, \ldots$ and $\mathrm{N}+\mathrm{P} \geq 0$ \\
\hline & Quadratic smile & $\begin{array}{ll}Y_{\text {slit }}{ }^{2} & \\
Y_{\text {slit }}{ }^{2} X_{\lambda} & Y_{\text {slit }}{ }^{2} X_{\text {slit }} \\
\text { (etc.) }\end{array}$ & $\begin{array}{l}M=1 \\
\text { Quadratic smile }(N, P)=(0,0) \\
+ \text { its linear varitions }(N, P)=(1,0) \text { and }(N, P)=(0,1) \text {. }\end{array}$ \\
\hline & $4^{\text {th }}$ order smile & $\begin{array}{l}Y_{\text {slit }}{ }^{4} \\
Y_{\text {slit }}{ }^{4} X_{\lambda} \quad Y_{\text {slit }}{ }^{4} X_{\text {slit }} \\
\text { (etc.) }\end{array}$ & $\begin{array}{l}M=2 \\
4^{\text {th }} \text { order smile }(N, P)=(0,0) \\
+ \text { its linear variations }(N, P)=(1,0) \text { and }(N, P)=(0,1) \text {. }\end{array}$ \\
\hline & Higher-order smile & $\begin{array}{l}\left(Y_{\text {slit }}\right)^{2 M} \\
\left(Y_{\text {slit }}\right)^{2 M} X_{\lambda} \quad\left(Y_{\text {slit }}\right)^{2 M} X_{\text {slit }} \\
\text { (etc.) }\end{array}$ & $\begin{array}{l}M=3,4 \text {, etc. }(N, P)=(0,0) \text { and linear variations } \\
(N, P)=(1,0) \text { and }(N, P)=(0,1) \text {. } \\
\text { Usually negligible. }\end{array}$ \\
\hline $\begin{array}{l}\text { Higher order } \\
\text { distortions } \\
\text { along Y }\end{array}$ & General form & $\left(Y_{\text {slit }}\right)^{2 M+1}\left(X_{\lambda}\right)^{N}\left(X_{\text {slit }}\right)^{P}$ & $\begin{array}{l}\text { with } \mathrm{M}=1,2,3, \ldots \text { and } \mathrm{N}+\mathrm{P} \geq 0 \text {. } \\
\text { All contributors are usually negligible. }\end{array}$ \\
\hline
\end{tabular}

Table 5. All possible distortions in systems with planar symmetry. The systems can be of any kind (reflective, refractive, catadioptric) as long as they have a plane of symmetry $(X, Z)$. Contributors with $P \geq 2$ are negligible since $X_{\text {slit }}$ is already very small.

\subsection{Collimator-less spectrometer designs}

\section{Compact collimator-less spectrometers}

Dispersive imaging spectrometers for space applications have been usually designed based on a traditional architecture: slit - collimator - disperser (prism or grating) - imager - detector. The Spectrolite family developed at TNO also used this approach with additionally freeform mirrors for reduced volume and improved performance.

In this section we present a new design possibility where a spectrometer layout without collimator is used. The resulting architecture is: slit - grating - imager - detector, the grating being directly illuminated with a diverging beam coming from the slit. This collimator-less configuration is usually avoided as the grating then generates strong non-rotationally symmetric aberrations. The use of freeform mirrors makes it possible to correct the strong aberrations and allows the collimator-less configuration. This type of design approach, where freeform mirrors are used in systems which would not work with classical optics, makes use of the full capabilities of freeform surfaces.

Two collimator-less spectrometers were obtained, with 1 and 2 freeform mirrors. A spectral range $400 \mathrm{~nm}-500 \mathrm{~nm}$ was selected, as required for $\mathrm{NO}_{2}$ remote sensing. Then, efforts were done to reach a spectral resolution of $0.5 \mathrm{~nm}$ and to maximize the etendue. If the obtained designs did not reach the targeted requirements (which are usually met with 4 freeform mirrors) the achieved performances are excellent and illustrate the capabilities of this design technique. The characteristics of the two designs are shown in Table 6 and compared to an example of Spectrolite spectrometer designed for $\mathrm{NO}_{2}$ remote sensing. 


\begin{tabular}{|l|c|c|c|}
\hline Spectrometer & $\begin{array}{c}\text { 1-mirror } \\
\text { collimator-less }\end{array}$ & $\begin{array}{c}\text { 2-mirror } \\
\text { collimator-less }\end{array}$ & Spectrolite \\
\hline Number of freeform mirrors & 1 & 2 & 4 \\
\hline Spectral range & $400-500 \mathrm{~nm}$ & $400-500 \mathrm{~nm}$ & $400-500 \mathrm{~nm}$ \\
\hline Spectral resolution & $1 \mathrm{~nm}$ & $1 \mathrm{~nm}$ & $0.5 \mathrm{~nm}$ \\
\hline Slit length & $14 \mathrm{~mm}$ & $20 \mathrm{~mm}$ & $20.89 \mathrm{~mm}$ \\
\hline Slit width & $0.144 \mathrm{~mm}$ & $0.144 \mathrm{~mm}$ & $0.151 \mathrm{~mm}$ \\
\hline Pupil shape assumption & circular & rectangular & rectangular \\
\hline F\# at slit & 8 & 8 & 5 (spatial) \\
\hline Relative etendue \\
(geometrical, excluding \\
spectral resolution)
\end{tabular}

Table 6. Compared characteristics and performance of 1-mirror and 2-mirror collimator-less spectrometers with an example of Spectrolite design.

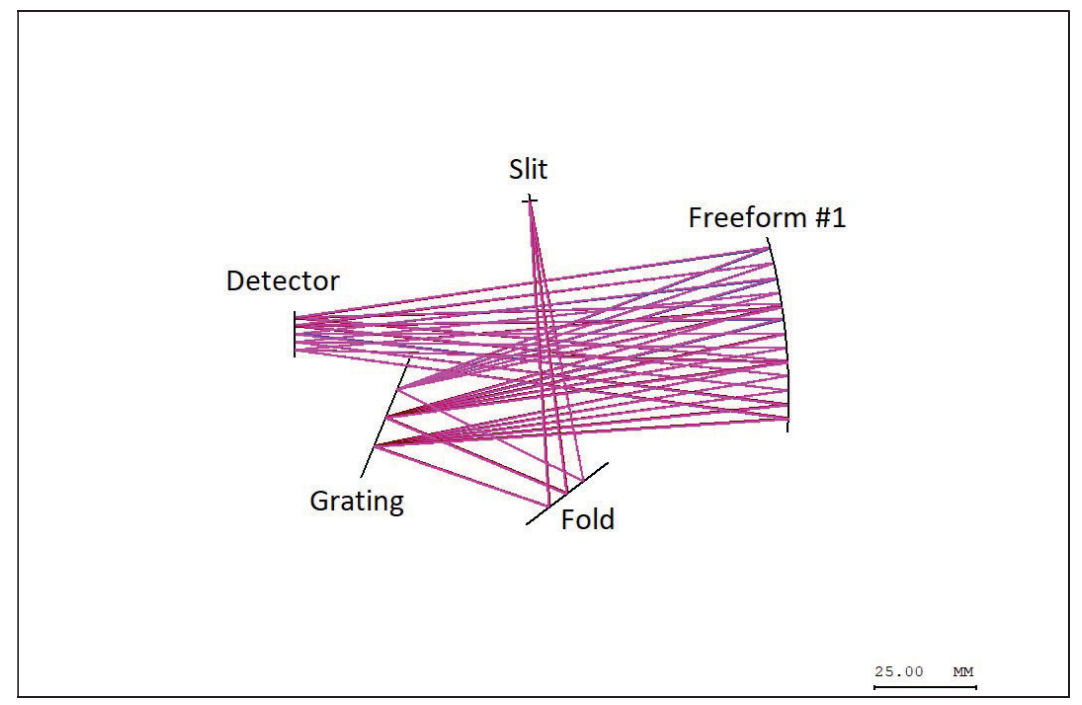

Figure 16. 1-mirror collimator-less spectrometer. 


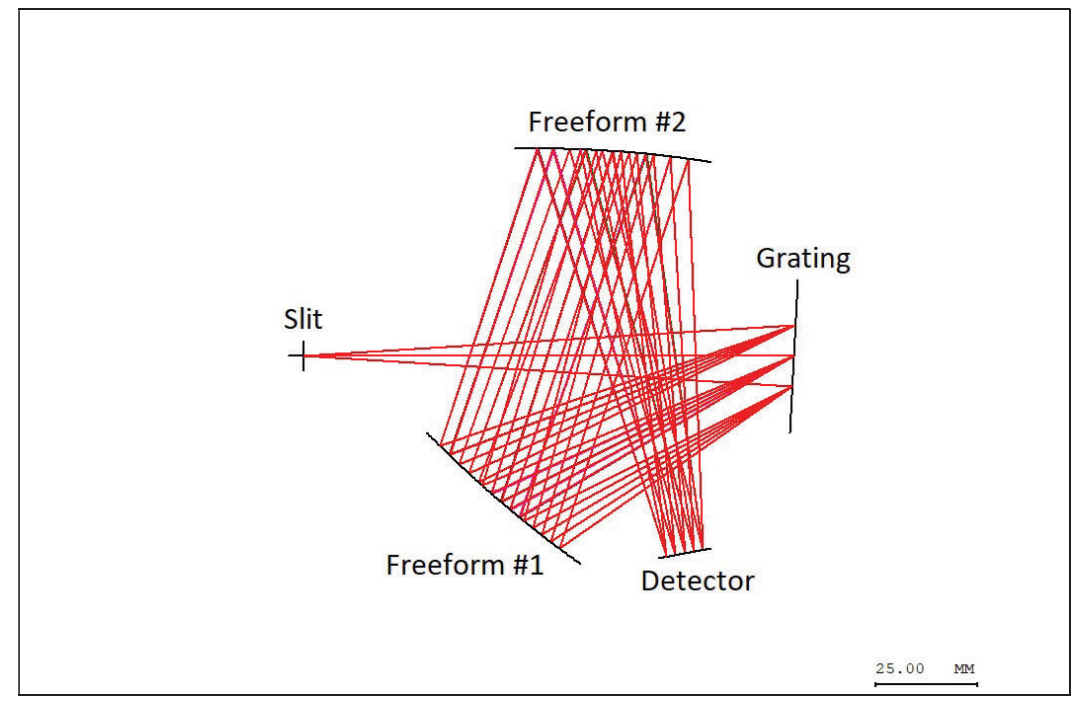

Figure 17. 2-mirror collimator-less spectrometer.

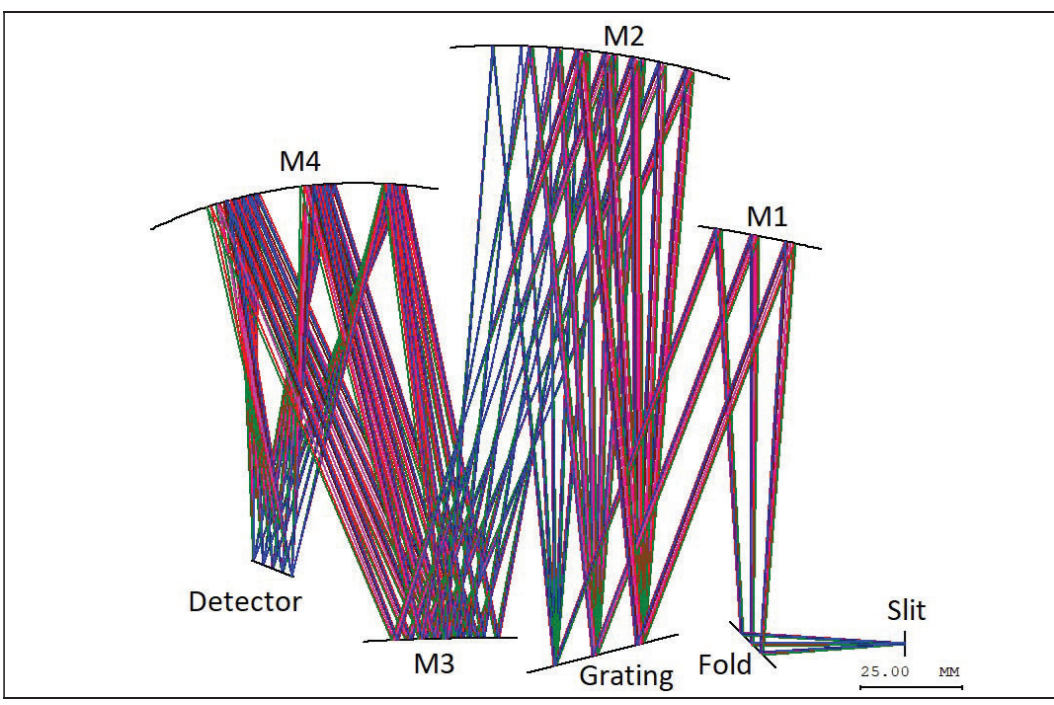

Figure 18. Spectrolite design, with 4 freeform mirrors. Same scale as Figures 16 and 17. The scale bar on the lower left part is $25 \mathrm{~mm}$ long (same as for figures 16 and 17).

It is worth noting that the 2 collimator-less designs were entirely calculated analytically, with only a final optimization of the high order freeform terms in CodeV. The benefit of using an analytical calculation comes from the reduced number of mirrors in a complex system. To correct all aberrations nearly all degrees of freedom must be used and the solution space is only a small part of the available parameter space. It becomes then challenging to find the solutions with simple optimization of mixed paraxial and freeform parameters. The aberration theory discussed in the previous sections [1,2] was extended to also account for flat reflective diffraction gratings, and used to find all the necessary conditions for aberration cancellation. Thanks to the obtained relationships, the few remaining degrees of freedom were identified, allowing to modify the designs very quickly. 


\section{$\underline{\text { Smile and keystone free collimator-less spectrometers }}$}

Simultaneous correction of smile and keystone is very challenging to achieve in freeform spectrometers with 2 or 3 mirrors in the imager. The aberration theory indicates that a 4-mirror imager is able to achieve a simultaneous correction under much more relaxed conditions. Using the collimator-less design approach, it is possible to correct smile and keystone simultaneously without increasing the number of mirrors in a 4-mirror spectrometer.

We present below a 4-mirror collimator-less spectrometer design that has the same characteristics as the Spectrolite spectrometer presented earlier: same spectral range $(400-500 \mathrm{~nm})$, spectral resolution $(0.5 \mathrm{~nm})$, slit and pupil dimensions, and is sized for the same detector. The obtained layout has also the same amount of freeform mirrors as the Spectrolite design, with the difference that they are now all located in the imager.

While the Spectrolite design has a very small (sub-pixel) keystone, its smile is limited by the linear variation of smile across the image which is very hard to correct. With the collimator-less design approach it was possible to correct both keystone and smile simultaneously including the smile linear variation across the image. The obtained design is approximately $1.5 x$ larger in volume than the Spectrolite spectrometer, partly due to the fact that mirrors are larger in the imager (since the field is then 2-dimensional) than in the collimator, and partly due to the fact that we targeted a better performance with smaller spot sizes.

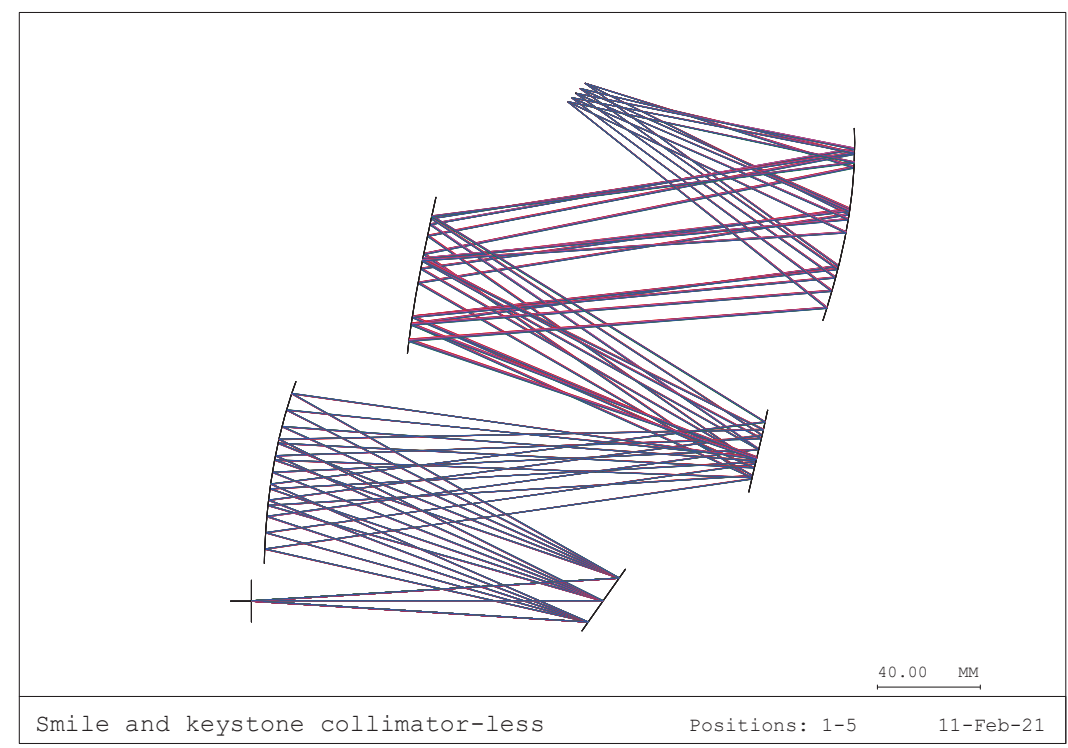

Figure 19. 4-mirror collimator-less spectrometer corrected for both smile and keystone. The scale bar on the lower left part is $40 \mathrm{~mm}$ long.

\begin{tabular}{|c|c|c|}
\hline Spectrometer & Collimator-less 4 mirrors & Spectrolite \\
\hline Max RMS spot diameter & $13.4 \mu \mathrm{m}$ & $19.8 \mu \mathrm{m}$ \\
\hline Smile & $5.3 \mu \mathrm{m}$ & $134.9 \mu \mathrm{m}$ \\
\hline Keystone & $1.9 \mu \mathrm{m}$ & $3.3 \mu \mathrm{m}$ \\
\hline
\end{tabular}

Table 7. Compared performance of a Spectrolite spectrometer and the 4-mirror collimator-less spectrometer. 


\section{SUMMARY AND OUTLOOK}

This paper summarizes some recent progress achieved at TNO on the design of freeform reflective systems. Designs with freeform mirrors are promising and allow to achieve an improved performance and/or a reduced size. To gain more understanding in this type of systems, efforts have been initiated a few years ago at TNO to develop a new theory that describes and predicts the aberrations of anamorphic freeform mirror systems with planar symmetry (commonly called "off-axis"). The obtained theory was recently published $[1,2]$ and fills a gap in the current knowledge on optical systems. It was also extended for flat reflection gratings, opening a way towards a full mathematical description of freeform mirror spectrometers.

This theory is useful to get a better understanding of the freeform design landscape (e.g. on the role of the design degrees of freedom) and helps to find better starting points for optimization. The insights gained also allow to understand how to tackle some performance problems such as the correction of distortions or to explore new design techniques. In this paper we have reported several new optical designs: 2-mirror telescopes for slit spectrometers corrected for spatial smile, 1-mirror and 2-mirror compact collimator-less spectrometers, as well as 4-mirror collimator-less spectrometers simultaneously corrected for smile and keystone.

Further work is ongoing. A matrix formalism was developed, to describe freeform multi-mirror systems and predict their aberrations up to an arbitrary order, which will be presented later this year at the IODC conference [16].

\section{REFERENCES}

[1] J. Caron and S. Bäumer, "Aberrations of plane-symmetrical mirror systems with freeform surfaces. Part I: generalized ray-tracing equations," J. Opt. Soc. Am. A 38, 80-89 (2021).

[2] J. Caron and S. Bäumer, "Aberrations of plane-symmetrical mirror systems with freeform surfaces. Part II: closed-form aberration formulas," J. Opt. Soc. Am. A 38, 90-98 (2021).

[3] D. Nijkerk, B. van Venrooy, P. Van Doorn, R. Henselmans, F. Draaisma, and A. Hoogstrate "The TROPOMI Telescope", Proc. SPIE 10564, International Conference on Space Optics (ICSO) 2012.

[4] R. Jansen, J.P.R. Day, R. Vink, J. de Vreugd, E.R.J. van Beekum, L.W. van der Laan, A.C.A. van 't Hof, W.L.M. Gielesen, and J. Koehler "Design and first light of the Sentinel-5 UV1 spectrometer optics", Proc. SPIE 11151, Sensors, Systems, and Next-Generation Satellites XXIII, 111510Q (10 October 2019).

[5] J. Koehler, R. Jansen, et al: "SENTINEL 5 instrument and UV1 spectrometers subsystem optical design and development", to be presented at the International Conference on Space Optics - ICSO 2020.

[6] D.Nijkerk, A.Meskers, B.Kruizinga, M.Gruber, N.Doelman, C.Duque, A Wide Field of View Telescope for Multi-Beam Optical Communication, Poster presented at: TNO Optical SatCom Day, November 2019.

[7] M. David Nijkerk, J. Michael Gruber, and B. Boonacker "Freeform optics design tool for compact spectrometers", Proc. SPIE 11180, International Conference on Space Optics - ICSO 2018, 1118022 (12 July 2019).

[8] D. Korsch, Reflective Optics (Academic, 1991).

[9] S. Chang, "Linear astigmatism of confocal off-axis reflective imaging systems with N-conic mirrors and its elimination," J. Opt. Soc. Am. A 32, 852-859 (2015).

[10] J. R. Rogers, "Origins and fundamentals of nodal aberration theory," in Optical Design and Fabrication Congress 2017 (IODC, Freeform, OFT) (2017).

[11] K. Fuerschbach, J. P. Rolland, and K. P. Thompson, "Theory of aberration fields for general optical systems with freeform surfaces," Opt. Express 22, 26585-26606 (2014).

[12] L. B. Moore, A. M. Hvisc, and J. Sasian, "Aberration fields of a combination of plane symmetric systems," Opt. Express 16, 15655-15670 (2008). L. B. Moore, A. M. Hvisc, and J. Sasian, "Aberration fields of a combination of plane symmetric systems: erratum," Opt. Express 17, 15390-15391 (2009).

[13] M. Tessmer, "Absence of a perfect intermediate reference wavefront for anamorphic systems," J. Opt. Soc. Am. A 37, 1423-1427 (2020).

[14]J.Rietjens, J.Campo, M.Smit, R.Winkelman, R.Nalla, J.Landgraf, O.Hasekamp, M.Oort, A.Van Amerongen, "Optical and system performance of SPEXone, a multi-angle channeled spectropolarimeter for the NASA PACE mission”, to be presented at the International Conference on Space Optics - ICSO 2020. 
[15] J. Caron, B. Sierk, J.-L. Bezy, A. Loescher, Y. Meijer, "The CarbonSat candidate mission: radiometric and spectral performances over spatially heterogeneous scenes", Proc. SPIE 10563, International Conference on Space Optics (ICSO) 2014.

[16] J.Caron, T.Ceccotti, S.Bäumer, "Progress in aberration theory for freeform off-axis mirror systems", invited paper, to be presented at the International Optical Design Conference - IODC 2021. 